Scott, R. A., \& Barber, B. L. (2020). Drinking among friends: The role of personality in links between online exposure to peer drinking and adolescent alcohol use. Cyberpsychology: Journal of Psychosocial Research on Cyberspace, 14(4), Article 5. https://doi.org/10.5817/CP2020-4-5

\title{
Drinking Among Friends: The Role of Personality in Links Between Online Exposure to Peer Drinking and Adolescent Alcohol Use
}

\author{
Riley A. Scott \& Bonnie L. Barber
}

School of Applied Psychology, Griffith University, Brisbane, Queensland, Australia

\begin{abstract}
The roles of conscientiousness and excitement seeking were investigated in the relationship between exposure to peer alcohol use online and adolescent alcohol consumption. It was hypothesized that higher levels of perceived peer alcohol use online would be associated with reports of higher adolescent alcohol consumption. Additionally, it was proposed that the relationship between perceived levels of peer alcohol use online and individual alcohol consumption would be stronger for adolescents lower in conscientiousness, and higher in excitement seeking, than it was in more conscientious, and less excitement-seeking students. Control variables included gender, pubertal timing, frequency of social networking site use, social networking site investment, and in-person peer alcohol norms. Data were collected through a cross-sectional survey from a sample of 1,018 students $(M=16.45$ years old, $S D=0.32$ years). Higher levels of in-person peer drinking norms and social networking site alcohol exposure were associated with higher adolescent drinking. Furthermore, excitement seeking significantly moderated the relationship between social networking site alcohol exposure and alcohol use. Participants reporting higher excitement seeking appeared more susceptible to online alcohol exposure than those reporting lower excitement seeking. The current study contributes to understandings of adolescent drinking by demonstrating personality differences in adolescent susceptibility to online alcohol consumption norms.
\end{abstract}

Keywords: Adolescence; alcohol use; personality; social media; social networking sites

\section{Introduction}

Adolescent alcohol consumption is a risk behavior with high social prevalence (Australian Institute of Health and Welfare, 2011). Recent reports suggest that risky adolescent drinking increases with age, and that 76\% of 17-yearolds report consuming alcohol in the past year (Guerin \& White, 2018; Kann et al., 2018). Given its applied importance through social consequences and potential for physical and developmental harm, research on the relationship between exposure to alcohol use and adolescent alcohol consumption is extensive (Kandel \& Andrews, 1987). In-person exposure to, and high estimates of, peer alcohol consumption are strongly associated with both higher intentions to consume alcohol and higher, more frequent alcohol consumption and binge drinking (Borsari \& Carey, 2001; Clapp \& McDonnell, 2000; Elmore et al., 2016). Social or self-comparisons made through interpretation of peers' behaviors have the power to influence adolescents' thoughts and subsequent behavior through the communication of descriptive peer norms (Beullens \& Vandenbosch, 2016; Chou \& Edge, 2012). Descriptive norms can influence behavioral intentions and participation in risk taking, as adolescents often contemplate the normative behaviors of peers when deciding whether to engage in risky behaviors themselves (Litt \& Stock, 2011). The Theory of Normative Social Behavior posits that normative beliefs predict alcohol consumption, with descriptive norms significantly contributing to intentions to consume alcohol (Rimal \& Real, 2005). 
Peer influence peaks in adolescence and may be amplified in online environments. The high prevalence of social networking site use as complementary or alternative to in-person or offline socialization, especially via mobile devices, means that peers' attitudes and behaviors are readily available, and may be viewed by adolescents at any time, and in any setting (Anderson \& Jiang, 2018; Huang et al., 2014; Lenhart, 2015). Feelings of positive engagement with social networking sites may heighten the use and perceived importance of social networking sites for social connection or motivate users to increase their investment in social networking platforms (Vernon et al., 2017). However, past research has highlighted potential disadvantages of investment in social networking sites, such as an association between social networking site investment and use and heightened vulnerability to the influences of peer modelling via social media (Huang et al., 2014). Despite this, few studies have investigated whether peer dynamics in the modelling of adolescent drinking transmit online via social networking sites.

As perceived peer norms in-person (or offline) predict adolescent drinking, it follows that exposure to peer alcohol use on social networking sites is also crucial to consider, given the common use of social networking sites by adolescents (Lenhart, 2015). For example, recent figures show that $95 \%$ of adolescents aged 13 to 17 own or have access to a smartphone, and 89\% report being online "at least multiple times per day" (Anderson \& Jiang, 2018, p. 8). The online network is a place to see both the prevalence of a behavior, and peers' implied acceptance of it. Normative information about peer alcohol use develops as adolescents extrapolate friends' behaviors and attitudes from what they see about the prevalence of online alcohol-related posts, and their peers' reactions to them (Rimal \& Real, 2005). Previous research has demonstrated that $85.3 \%$ of teenage boys' Facebook profiles contained visual and verbal references to alcohol use, the majority of which were framed positively (Beullens \& Schepers, 2013; Egan \& Moreno, 2011). A study by Fournier and Clarke (2011) further suggested that having more alcohol-related content on one's Facebook profile was associated with higher frequency and quantity of alcohol use. Litt and Stock (2011) revealed that adolescents exposed to older peers' alcohol use via social networking sites reported higher perceived peer consumption, more positive alcohol use attitudes, and reduced perceived vulnerability to consequences of alcohol consumption. Two additional studies recently demonstrated that higher exposure to alcohol-related posts online was predictive of greater descriptive and injunctive norms about peer alcohol consumption, future drinking intentions, and reported alcohol use (Beullens \& Vandenbosch, 2016; Stoddard et al., 2012). Further, S. C. Boyle and colleagues (2016) demonstrated that exposure to peers' alcoholrelated content on social media significantly predicted college students' alcohol use six months later. This predictive effect persisted when controlling for close friends' offline drinking behavior (S. C. Boyle et al., 2016). Such research highlights the need for further consideration of the use of social networking sites in the exposure to, and development of, peer norms surrounding alcohol use.

\section{Personality}

Although previous research has demonstrated the existence of a link between online exposure to peer alcohol use and alcohol consumption, this relationship is not the same for all adolescents (Borsari \& Carey, 2001). The Differential Susceptibility to Media Effects Model (DSMM; Valkenburg \& Peter, 2013) outlines dispositional features of media users that might buffer or exacerbate media effects, including gender, motivation and personality. Individual differences in personality can reliably predict a person's interpersonal behaviors, alongside their emotions, experiences, motivations, and attitudes (McCrae \& John, 1992). The five-factor model of personality includes the traits of conscientiousness, extraversion, openness to experience, agreeableness, and neuroticism (McCrae \& John, 1992). Of the five factors, conscientiousness and extraversion have shown the most promise in relationship to health risk behavior and drinking. Therefore, in order to develop a more targeted understanding of individual differences in the relationship between exposure to alcohol-related content on social networking sites and alcohol consumption, these two traits were included in the current study.

Conscientiousness and extraversion were selected for inclusion in the present study as previous research has consistently demonstrated significant effects of these personality traits for engagement in health risk behaviors (Bogg \& Roberts, 2004; Hong \& Paunonen, 2009; Raynor \& Levine, 2009). The first trait of interest, conscientiousness, contains sub-facets including self-discipline, competence, order, and deliberation (De Fruyt et al., 2000). People high in conscientiousness are often described as organized, careful, and responsible, whereas their less conscientious counterparts may be described as reckless, irresponsible, and absent-minded (G. J. Boyle et al., 2008). The second trait, extraversion, incorporates five sub-facets including excitement seeking, which was examined in the current study (De Fruyt et al., 2000; Klimstra, 2013). Individuals reporting high excitement seeking 
often report feelings of disinhibition, boredom susceptibility (Glazer et al., 2010), and a greater willingness to accept both physical and social risks associated with potentially dangerous experiences, than their lower excitement seeking counterparts (Kim \& Kim, 2012).

Research investigating the effects of the five-factor model of personality (McCrae \& John, 1992) on adolescent alcohol consumption has previously highlighted individual differences in both conscientiousness and excitement seeking as predictive of adolescents' drinking. Adolescents lower in conscientiousness engage more often in risky substance use, whereas adolescents higher in conscientiousness are less likely to drink, and begin drinking at a later age than their less conscientious peers (Hagger-Johnson et al., 2012; McCabe et al., 2016). Past research has also revealed a relationship between excitement seeking and alcohol consumption; higher excitement seeking has previously explained the positive relationship between extraversion and drinking behaviors (Hong \& Paunonen, 2009). Higher excitement seeking and increased perceptions of peer alcohol use also significantly predict young adults' alcohol consumption (Glazer et al., 2010). These well-documented links between personality and alcohol use guided the decision to explore conscientiousness and excitement seeking in links between social networking site alcohol exposure and adolescent alcohol use. Existing research also suggests that conscientiousness and extraversion are each related to social networking site use. Adolescents' reporting both low and high conscientiousness have demonstrated higher social networking site use relative to those with moderate levels of conscientiousness (Marino et al., 2016; Wilson et al., 2010). Further, higher extraversion has previously predicted higher frequency of social networking site use, due to extroverts' drive for social connection, stimulation, and activity (Marino et al., 2016, Wilson et al., 2010).

\section{Personality, Social Networking Site Alcohol Exposure, and Alcohol Use}

The influence of personality on adolescents' susceptibility to peers' drinking behavior norms online has rarely been examined in research. In Glazer and colleagues' (2010) study, sensation seeking did not moderate the relationship between offline peer norm perceptions and alcohol use, but significantly moderated the relationship between believability of peer norm messages and alcohol use. Beullens and Vandenbosch (2016) controlled for excitement seeking when examining the relationship between social networking site use, peer norms, and intentions to consume alcohol. Excitement seeking did contribute significantly to predicting alcohol attitudes, norms, and intentions to drink in the presence of online alcohol-related peer norms. Geusens and colleagues (2019) reported that adolescents who shared alcohol-related content on social networking sites were more susceptible to engaging in alcohol consumption when higher in extraversion and lower in conscientiousness. In considering such previous research, it becomes apparent that further investigation of personality as a moderator of the link between online peer norms and adolescent drinking behaviors is needed. This is particularly important considering the potential for conscientiousness and excitement seeking to buffer against or exacerbate the effects of exposure to online alcohol-related posts, respectively.

The present study therefore aims to increase understanding about adolescent alcohol use, by investigating whether individual differences in adolescents' conscientiousness and excitement seeking moderate the link between social networking site peer alcohol exposure and alcohol consumption. Based on evidence for links to alcohol use and online exposure to alcohol, this study will control for gender (Beullens \& Schepers, 2013; Egan \& Moreno, 2011), pubertal timing (Modecki et al., 2014; Stattin et al., 1989), offline peer drinking norms (S. C. Boyle et al., 2016; Kandel \& Andrews, 1987), social networking site frequency, and social networking site investment (Australian Communications and Media Authority, 2013). More frequent use of social networking sites may be associated with higher exposure to positive alcohol messages, which in turn, may underpin adolescents' positive attitudes and peer norms towards alcohol use.

Based on preliminary research findings suggesting a possible link between exposure to peer alcohol use on social networking sites and adolescent alcohol consumption, as well as the clear potential for conscientiousness and excitement seeking to moderate the effects of exposure to online alcohol-related posts, two hypotheses were proposed:

H1: Higher levels of perceived peer alcohol use online would be associated with reports of higher adolescent alcohol consumption. 
H2: The relationship between perceived levels of peer alcohol use online and individual alcohol consumption would be stronger for less conscientious students than for more conscientious students, and for higher excitement seeking students than for lower excitement seeking students.

\section{Method}

\section{Participants}

Participants completed the 2013 Youth Activity Participation Survey of Western Australia (YAPS); a longitudinal study of Australian adolescents' organized activity participation, leisure time use, social contexts, and individual attributes and well-being. Participants were 1,018 Year 11 students (517 females) from 36 high schools (73.9\% metropolitan districts, $26.1 \%$ regional). The number and type of schools (government, non-government) selected within each district was determined by high school student enrolment rates. Most students (90.9\%) reported 'yes' to "Have you ever created your own profile online that others can see, like on a social networking site like Facebook, Myspace, or Bebo"; only these participants were included in analyses. Of the 925 students included in the analyses, 470 (50.8\%) were female. The mean age of participants was 16.45 years old (SD $=0.32$ years). Facebook was reported as the most commonly used social networking platform, with $86.8 \%$ of the included participants indicating that Facebook was the profile they updated most often.

\section{Materials and Procedure}

Prior to data collection, the research was granted approval and ethical clearance by the University Human Research Ethics Committee. Students in groups of 25 to 30 completed the survey within a 30- to 40-minute timeframe, using either iPads or paper-and-pencil, under researcher-supervised conditions at all schools, often in a classroom, library or meeting room. The confidential surveys were completed as part of a longitudinal study in which both students and their parents were required to provide informed and written consent before participation. Students were assured that their answers to survey questions were confidential and would not be shared with their teachers, school, or parents. All participating students were offered entry into an immediate lottery for prizes at both the school level (potential prizes included vouchers or sporting memorabilia) and in an overall prize draw (e.g., guitar or iPod).

\section{Measures}

\section{Pubertal Timing}

Pubertal timing was measured with one item that asked participants when they believed their physical development began, in comparison to same-age peers. Responses were chosen from a five-point scale $(1=$ Much later, to $5=$ Much earlier). Higher scores are indicative of relatively earlier pubertal timing.

\section{Social Networking Site Frequency}

Adolescents' frequency of social networking site use was measured with two items. One YAPS-specific item asked: "How often do you visit your social networking site?" One item adapted from Lenhart and Madden (2007) asked participants: "About how often do you visit your profile?" Responses for both items were recorded on a sevenpoint scale $(1=$ Never, to $7=$ Several times $a$ day). Higher scores indicated greater frequency of social networking site use. The mean was computed, with scale reliability confirmed $(a=.64)$.

\section{Social Networking Site Investment}

Two items measuring social networking site investment were adapted from Ellison and colleagues' (2007) 'Facebook Intensity Scale'. Participants were asked to indicate their level of agreement for the following two statements: "Facebook/Myspace/Bebo has become part of my daily routine", and; "I feel out of touch when I haven't logged on to Facebook/Myspace/Bebo". Responses were recorded on a five-point scale $(1=$ Completely 
disagree to 5 = Completely agree). Higher scores reflect greater investment in social networking site use. The mean was computed and demonstrated good internal consistency $(a=.78)$.

\section{Offline Peer Alcohol Norms}

Peer alcohol use perceptions were measured with four items across three participation contexts: friends in sports, friends in non-sport organized activities, and friends not involved in either sports or activities. For those who participated in sports and/or other organized activities, participants were asked to indicate the proportion of their friends in their sports or activities who regularly drank alcohol ("What proportion of your peers [in this sport/activity] regularly drink alcohol?" One question per context). Further, all participants involved in sports and/or activities reported the proportion of their friends not involved in either activity who regularly drank alcohol. Finally, participants who were not involved in either sports or activities responded to a global item by reporting the proportion of their friends who regularly drank alcohol. Thus, participants responded to between one and three questions, depending upon their involvement in sport and non-sport extracurricular activities. A mean of up to three items was computed to represent offline peer drinking norms across contexts. All responses were recorded on a 5-point scale, from $1=$ None to $5=$ All. Higher scores indicate perceptions of higher proportions of offline peers who drank.

\section{Social Networking Site Alcohol Exposure}

The Social Networking Site Alcohol Exposure measure from Pegg et al. (2018) was included to assess adolescents' online exposure to the alcohol consumption of peers. The item was "About how often in the last 6 months have your friends posted pictures, updates, or wall posts that showed or talked about them drinking alcohol?" Participants were asked to circle one answer along an eight-point scale ( $1=$ None, to $8=31$ or more times). Higher scores indicate more peer-related alcohol exposure online.

\section{Alcohol Use}

Participants' alcohol consumption across the past six months was measured with three items adapted from Fredricks and Eccles' (2005) Alcohol Use scale. Participants reported how often they had drunk alcohol, had more than five alcoholic drinks on one occasion, and been drunk in the past six months. Responses were recorded along an eight-point scale $(1=$ None, to $8=31$ or more times), with high scores indicating more frequent alcohol consumption. A mean was computed, for which internal reliability was confirmed $(a=.95)$.

\section{Personality}

Two measures of personality were used to assess conscientiousness and excitement seeking. A seven-item conscientiousness scale, and five-item excitement seeking scale, were derived from the Revised NeuroticismExtraversion-Openness Personality Inventory (NEO-PI-R; Costa \& McCrae, 1992). Participants answered the twelve personality items along a five-point agreement scale ( $1=$ Strongly disagree, to $5=$ Strongly agree $)$. Participants' mean responses to the conscientiousness and excitement seeking items were computed. Example conscientiousness items include "I am always prepared", and "I avoid my responsibilities" (reverse scored). Excitement seeking items included statements such as "I love action", and "I am willing to try anything once". Scale reliability was confirmed for Conscientiousness $(\alpha=.69)$, and Excitement Seeking $(\alpha=.71)$.

\section{Data Analytical Approach}

Statistical analyses were run using SPSS version 24 and the Process macro (Hayes, 2013). First, we ran descriptive statistics and bivariate correlations to examine the relationships between all variables of interest. We then conducted a moderated multiple regression analysis to determine whether the association between Social Networking Site Alcohol Exposure and Alcohol Use was moderated by Conscientiousness or Excitement Seeking. In testing the assumptions prior to the analyses, it was noted that severe skew was present in terms of the dependent variable, Alcohol Use, and determined that no data transformation assisted in managing this violation. Due to the participants' age however, this skew was expected, because many of the underage adolescents 
reported no alcohol use. Multiple regression analyses have previously shown robustness despite distributional assumptions not being met with skewed alcohol use data (Neal \& Simons, 2007). The data were thus used without transformation, as a representation of real-world alcohol use patterns in adolescents. Finally, significant interaction terms in the analysis were examined by conducting simple slopes analyses in the Process macro (Hayes, 2013).

\section{Results}

Bivariate correlations and descriptive statistics are presented in Table 1. Relationships of weak to moderate strength were found between all variables. Importantly, participants who reported higher levels of both Offline Peer Alcohol Norms and Social Networking Site Alcohol Exposure also reported greater Alcohol Use. Excitement Seeking and Conscientiousness were positively and negatively associated with Alcohol Use, respectively. These correlations signify that participants higher in Excitement Seeking and lower in Conscientiousness reported more Alcohol Use. Social Networking Site Frequency, Social Networking Site Investment, and earlier Pubertal Timing were also significantly and positively correlated with Alcohol Use. Gender was not significantly correlated with Alcohol Use.

Table 1. Means, Standard Deviations (SD), and Pearson Correlations Amongst Measures of Gender, Pubertal Timing, Social Networking Site Frequency, Social Networking Site Investment, Offline Peer Alcohol Norms, Social Networking Site Alcohol Exposure, Conscientiousness, Excitement Seeking, and Alcohol Use $(N=819)$.

\begin{tabular}{|c|c|c|c|c|c|c|c|c|c|}
\hline & 1. & 2. & 3. & 4. & 5. & 6. & 7. & 8. & 9. \\
\hline 1. Gender ${ }^{\dagger}$ & - & & & & & & & & \\
\hline 2. Pubertal Timing ${ }^{\ddagger}$ & $-.072^{\star}$ & - & & & & & & & \\
\hline 3. SNS Frequency & -.002 & $.099 * *$ & - & & & & & & \\
\hline 4. SNS Investment & $-.092 * \star$ & $.072 *$ & $.464 * \star$ & - & & & & & \\
\hline 5. Offline Alcohol Norms & $.082 *$ & .050 & $.186^{\star \star}$ & $.199 * *$ & - & & & & \\
\hline 6. SNS Alcohol Exposure & .021 & .051 & $.190 * \star$ & $.266^{\star *}$ & $.487 * \star$ & - & & & \\
\hline 7. Conscientiousness & -.006 & .047 & $-.095 * \star$ & $-.144 * \star$ & $-.156 * \star$ & $-.080 *$ & - & & \\
\hline 8. Excitement Seeking & .046 & .014 & $.091 * *$ & $.154^{\star *}$ & $.191 * *$ & $.126^{\star *}$ & .050 & - & \\
\hline 9. Alcohol Use & .040 & $.084 *$ & $.182^{\star \star}$ & $.211 * \star$ & $.538 * \star$ & $.405^{\star \star}$ & $-.122 * \star$ & $.206 * \star$ & - \\
\hline Mean & & 3.03 & 5.42 & 2.96 & 2.19 & 3.82 & 3.51 & 3.69 & 2.02 \\
\hline$S D$ & & 0.95 & 1.27 & 1.22 & 1.05 & 2.33 & 0.52 & 0.66 & 1.53 \\
\hline
\end{tabular}

Note. ${ }^{\dagger}$ Gender: $0=$ Female, $1=$ Male ${ }^{\ddagger}$ Higher scores represent relatively earlier pubertal timing.

SNS $=$ Social Networking Site.

$\star_{p}<.05, * * p<.01$.

A moderated multiple regression analysis was conducted to determine whether the association between Social Networking Site Alcohol Exposure and Alcohol Use was moderated by Conscientiousness or Excitement Seeking. Alcohol Use was the dependent variable. Control variables entered at Step 1 were Gender, Pubertal Timing, Social Networking Site Frequency, and Social Networking Site Investment. Offline Peer Alcohol Norms, Social Networking Site Alcohol Exposure, Conscientiousness, and Excitement Seeking were entered at Step 2, followed by the interaction terms, "Alcohol Exposure × Conscientiousness", and "Alcohol Exposure $\times$ Excitement Seeking" at Step 3.

At Step 1, 6.1\% of the variance in adolescent Alcohol Use was explained by Gender, Pubertal Timing, Social Networking Site Frequency and Social Networking Site Investment, $F(4,814)=13.23, p<.001$ (see Table 2). Adolescents who reported more frequent social networking site use and higher social networking site investment, also reported higher levels of alcohol use. Gender and Pubertal Timing did not significantly contribute to the model. Step 2 was significant, and resulted in the explanation of an additional $27.4 \%$ of the variance in Alcohol Use, $F_{c h g}(4,810)=83.45, p<.001$. Offline Peer Alcohol Norms, Social Networking Site Alcohol Exposure, and Excitement Seeking significantly predicted alcohol use. Adolescents who reported higher perceptions of offline peer drinking, more peer-related alcohol exposure online, and higher excitement seeking, also reported higher levels of alcohol use. Conscientiousness did not significantly contribute to the model. The interaction terms entered at Step 3 of the analysis also made a significant unique contribution, adding 1.0\% of the variance explained 
in Alcohol Use, $F_{c h g}(2,808)=6.29, p=.002$. The Alcohol Exposure $\times$ Excitement Seeking interaction term was significant. The interaction between Alcohol Exposure $\times$ Conscientiousness did not contribute significantly to the model. In Step 3, the main effects of Social Networking Site Alcohol Exposure and Excitement Seeking were no longer significant. A total of $34.5 \%$ of the variance in Alcohol Use was explained by the final model, $F(10,808)=$ $42.61, p<.001$ (see Table 2).

Table 2. Summary of Moderated Multiple Regression Analysis, Testing the Effects of Conscientiousness and Excitement Seeking on the Association Between Social Networking Site Alcohol Exposure and Alcohol Use $(N=819)$.

\begin{tabular}{|c|c|c|c|c|c|}
\hline \multirow{2}{*}{ Variable } & \multirow{2}{*}{$B$} & \multirow{2}{*}{$S E(B)$} & \multicolumn{2}{|c|}{$95 \% \mathrm{Cl}(B)$} & \multirow{2}{*}{$\beta$} \\
\hline & & & Lower & Upper & \\
\hline \multicolumn{6}{|l|}{ Step 1} \\
\hline Constant & 0.34 & .28 & -0.20 & 0.89 & \\
\hline Gender $^{\dagger}$ & 0.19 & .11 & -0.02 & 0.39 & .06 \\
\hline Pubertal Timing ${ }^{\ddagger}$ & 0.11 & .06 & 0.00 & 0.22 & .07 \\
\hline SNS Frequency & 0.12 & .05 & 0.03 & 0.21 & $.09 *$ \\
\hline SNS Investment & 0.21 & .05 & 0.11 & 0.30 & $.17 * \star \star$ \\
\hline \multicolumn{6}{|l|}{ Step 2} \\
\hline Constant & -0.36 & .24 & -0.84 & 0.11 & \\
\hline Gender & 0.02 & .09 & -0.16 & 0.19 & .01 \\
\hline Pubertal Timing & 0.08 & .05 & -0.01 & 0.17 & .05 \\
\hline SNS Frequency & 0.04 & .04 & -0.04 & 0.12 & .04 \\
\hline SNS Investment & 0.06 & .04 & -0.03 & 0.14 & .04 \\
\hline Offline Alcohol Norms & 0.60 & .05 & 0.51 & 0.70 & $.41 * \star \star$ \\
\hline SNS Alcohol Exposure & 0.11 & .02 & 0.07 & 0.15 & $.17 * \star \star$ \\
\hline Conscientiousness & -0.12 & .09 & -0.29 & 0.05 & -.04 \\
\hline Excitement Seeking & 0.22 & .07 & 0.09 & 0.36 & $.10 * \star$ \\
\hline \multicolumn{6}{|l|}{ Step 3} \\
\hline Constant & -0.39 & .24 & -0.87 & 0.08 & \\
\hline Gender & 0.01 & .09 & -0.16 & 0.19 & .00 \\
\hline Pubertal Timing & 0.09 & .05 & -0.00 & 0.18 & .06 \\
\hline SNS Frequency & 0.04 & .04 & -0.04 & 0.11 & .03 \\
\hline SNS Investment & 0.06 & .04 & -0.02 & 0.15 & .05 \\
\hline Offline Alcohol Norms & 0.60 & .05 & 0.50 & 0.70 & $.41 * \star *$ \\
\hline SNS Alcohol Exposure & 0.11 & .02 & 0.07 & 0.15 & $.16 * \star \star$ \\
\hline Conscientiousness & 0.14 & .16 & -0.17 & 0.46 & .05 \\
\hline Excitement Seeking & -0.11 & .12 & -0.35 & 0.14 & -.05 \\
\hline Alcohol Exposure $\times$ Conscientiousness & -0.07 & .04 & -0.14 & 0.01 & -.10 \\
\hline Alcohol Exposure $\times$ Excitement Seeking & 0.08 & .03 & 0.03 & 0.14 & $.16^{\star \star}$ \\
\hline
\end{tabular}

Note. ${ }^{\dagger}$ Gender: $0=$ Female, $1=$ Male.${ }^{\ddagger}$ Higher scores represent relatively earlier pubertal timing. SNS $=$ Social Networking Site.

$\star p<.05, * * p<.01, * \star * p<.001$.

To probe the significant Alcohol Exposure $\times$ Excitement Seeking interaction, simple slopes analyses were conducted using the Process macro (Hayes, 2013) in SPSS for low, moderate, and high levels of Excitement Seeking. The points were created using a pick-a-point technique in which one standard deviation below the mean, the mean, and one standard deviation above the mean were used to represent low, moderate, and high levels of both Social Networking Site Alcohol Exposure and Excitement Seeking. A significant positive relationship was found between Social Networking Site Alcohol Exposure and Alcohol Use, at moderate $(B=.11, p<.001)$, and high $(B=$ $.16, p<.001)$, levels of Excitement Seeking, but not at low levels of Excitement Seeking $(B=.05, p=.07)$. This result indicates that for adolescents of moderate and high excitement seeking, more exposure to peer alcohol use via social networking sites was associated with significantly greater alcohol consumption. The association between 
Social Networking Site Alcohol Exposure and Alcohol Use was strongest for adolescents higher in Excitement Seeking, and not significant for those lower in Excitement Seeking (see Figure 1) ${ }^{1}$.

Figure 1. The Conditional Relationship Between Social Networking Site Alcohol Exposure and Alcohol Use (Controlling for Covariates) at Low, Moderate, and High Levels of Excitement Seeking ( $N=819)$. Error Bars Represent Standard Errors.

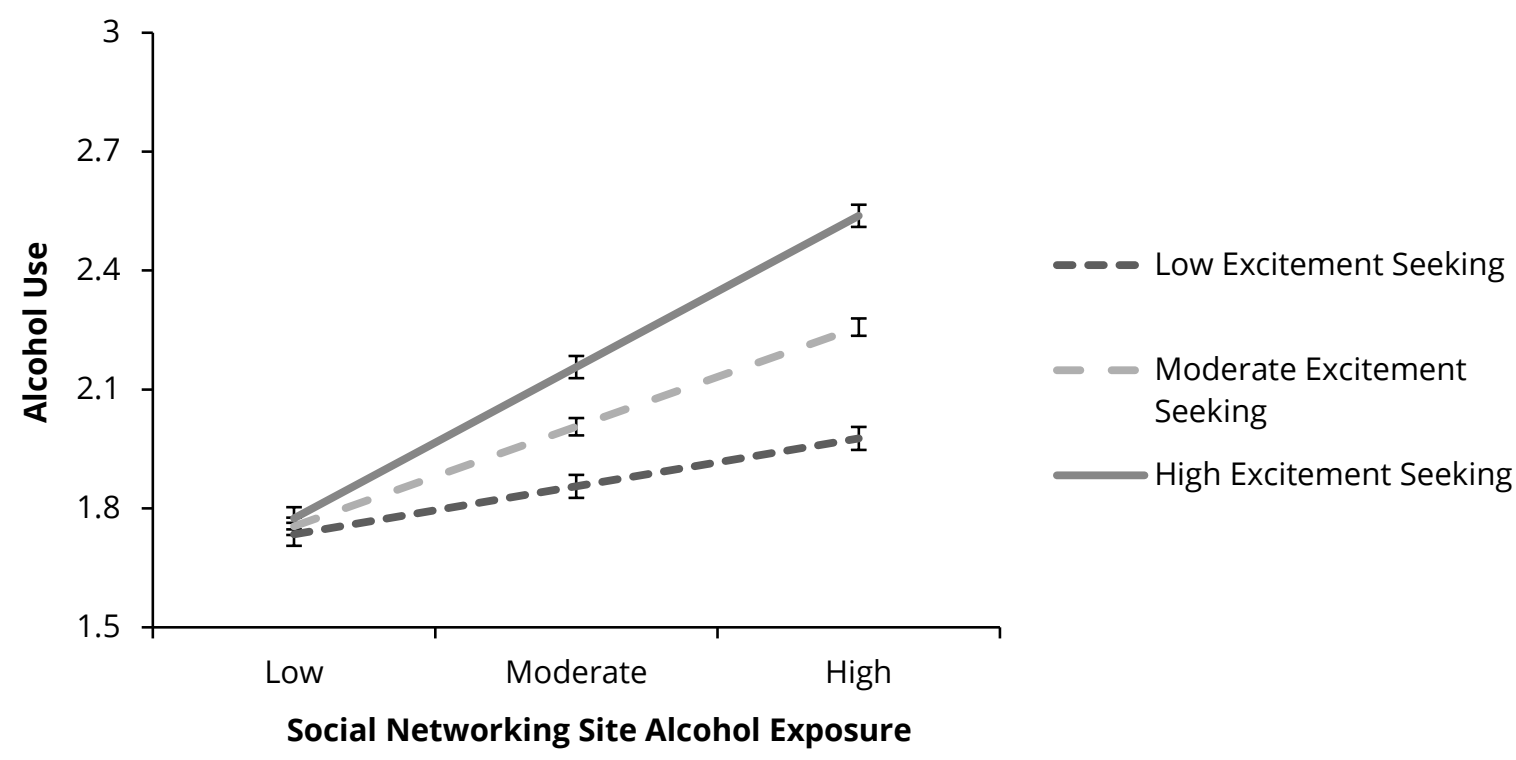

\section{Discussion}

Adolescents are differentially susceptible to peer influence, but personality is not well understood as a potential moderator of the link between online peer norms and adolescent alcohol use. In addressing this gap, the present study investigated whether individual differences in adolescents' conscientiousness and excitement seeking moderated the link between social networking site peer alcohol exposure and alcohol consumption. In support of the first hypothesis, more exposure to peer alcohol use via social networking sites was significantly associated with higher alcohol use, consistent with previous research (e.g. Geusens et al., 2019; Pegg et al., 2018). Further, social networking site alcohol exposure was significantly associated with higher alcohol use even when offline peer alcohol norms were controlled. The current finding also extends some previous research that considered intentions to use alcohol, with no measure of drinking behavior (Beullens \& Vandenbosch, 2016). The relationship between exposure to peer alcohol use and adolescent alcohol consumption is extensively documented (Beullens \& Vandenbosch, 2016; Clapp \& McDonnell, 2000; Kandel \& Andrews, 1987; Schroeder \& Prentice, 1998), and thus, it was not surprising that the strongest predictor of adolescent drinking in the current study was offline peer alcohol norms. However, despite this, our results highlight the importance of considering social networking site use as an additional source of exposure to peer alcohol use norms.

The second hypothesis, that adolescents higher in excitement seeking would be more susceptible to social networking site alcohol exposure, and report a stronger link between social networking site exposure and alcohol consumption than their peers lower in excitement seeking, was supported. The relationship between social networking site alcohol exposure and alcohol use was significant at moderate, and high levels of excitement seeking, with adolescents higher in excitement seeking having the greatest propensity to consume alcohol when exposed to peers' alcohol-related posts online. The limited amount of previous research examining the influence of excitement seeking (as a sub-facet of extraversion) in the relationship between exposure to peer norms and alcohol use is characterized by mixed results. In previous research, sensation seeking has not been found to moderate between perceptions of offline peer alcohol use norms and alcohol use (Glazer et al., 2010). However, for online social networking, when included as a control variable, excitement seeking demonstrated a significant influence on alcohol-related attitudes and intentions to consume alcohol in the presence of online peer norms (Beullens \& Vancenbosch, 2016). The current study extends previous research, by highlighting the importance of excitement seeking in moderating the relationship between adolescent social networking site alcohol exposure and alcohol use. This finding demonstrates a potential vulnerability for adolescents exhibiting particular 
personality traits facing online exposure to peer drinking. One explanation might be that people high in excitement seeking have been shown to report similarly high levels of disinhibition, boredom susceptibility, and an increased drive for involvement in novel and thrilling experiences (Glazer et al., 2010), perhaps leading to greater susceptibility to peer influence towards alcohol use.

Excitement seeking may have intensified the link between online alcohol exposure and adolescent drinking through increased drive for socialization seen in adolescents with higher levels of extraversion. Bekman et al. (2010) describe higher excitement seekers as more likely to attend to positive alcohol media messages, and more likely to develop anxiety regarding consequences of non-engagement in alcohol use as a social activity. Low levels of behavioral regulation seen in higher excitement-seeking individuals have also been associated with higher descriptive norms and drinking (Hustad et al., 2014). Such characteristics of excitement seeking may exacerbate the effects of online alcohol messages on alcohol consumption, making higher excitement-seeking adolescents more susceptible to the online exposure of peer alcohol use than their lower excitement-seeking counterparts.

The prediction that the relationship between perceived levels of peer alcohol use online and individual alcohol consumption would be stronger for less conscientious students than for more conscientious students was not supported. Conscientiousness demonstrated a relationship with alcohol consumption only at the bivariate level. When included in the regression analyses alongside excitement seeking however, conscientiousness did not significantly independently contribute to explaining adolescent alcohol use. Sub-facets of conscientiousness including self-discipline and deliberation, and their associated characteristics of self-control, constraint and responsibility, have previously demonstrated associations with alcohol use (Bogg \& Roberts, 2004; De Fruyt et al., 2000). One possible explanation for the lack of significant results for conscientiousness in the present study is that any moderating power of conscientiousness in the link between SNS peer-related alcohol exposure and adolescent drinking lies within its facets. Conscientiousness is also related to reporting fewer offline friends who drink, so some of its impact may be indirect through the broader role of protective peer affiliations. Future research investigating the role of sub-facets of conscientiousness or peer connections could explore this possibility.

The implications of this study of adolescent drinking behaviors may extend beyond contribution to theory. Firstly, there is potential for identifying at-risk (higher excitement seeking) adolescents, whereby monitoring and control of online content they see may buffer them against peer pressure to consume alcohol. Future research is needed to investigate the content of alcohol-related posts presented to adolescents online. Doing so could assist in expanding our limited understanding of what types of posts and content motivate underage adolescents to engage in drinking. Secondly, limiting adolescent susceptibility to the power of peer norms is crucial in reducing the impact that social networking site alcohol exposure has on alcohol consumption. Disputing positive attitudes and expectancies about alcohol consumption as they are developed through online exposure to peer alcohol use - through descriptive norms education - may reduce both the perceived pressure to drink, and the fears of negative social evaluation held by non-drinkers or adolescents emulating their peers' drinking behaviors (Schroeder \& Prentice, 1998). Norms education interventions have previously shown success in reducing both the perceptions of peer binge drinking as normative, and self-reported binge drinking levels (Haines \& Spear, 1996).

Although controlling for gender, pubertal timing, social networking site frequency, social networking site investment and offline peer alcohol norms was a strength of this study, there are limitations to be considered. First, the cross-sectional nature of the data does not allow for causality to be determined. With the measurement of all constructs at one time it is not only possible that exposure to peer alcohol use on social networking sites leads to an increase in alcohol use, but also that the converse might be true. Those who regularly consume alcohol may seek out more alcohol-related content online. However, it must be noted that determining directionality of the current associations between social networking site alcohol exposure, personality, and alcohol use through use of longitudinal data may prove difficult as reciprocal relationships are likely in considering alcohol norms and alcohol use (Drane et al., 2017; Geusens \& Beullens, 2017). Additional limitations within the current study include the use of self-report data in measuring all constructs, and the use of a one-item measure for social networking site alcohol exposure. Self-report data are consistently used in adolescent research, and were expected to be particularly useful in the current study, as consumption of alcohol is driven by normative beliefs and perceptions of peers' alcohol consumption, rendering the self-report views central to the processes of interest (Rimal \& Real, 2005). Further, the one-item Social Networking Site Alcohol Exposure measure in the current study demonstrates 
good content validity, and has significantly explained adolescent alcohol use in previous research (Pegg et al., 2018). The utility of this construct is further demonstrated as, in support of past research (S. C. Boyle et al., 2016), social networking site exposure to peers' alcohol-related content contributed to explaining adolescent drinking, over and above perceptions of offline peers' alcohol use. It must also be noted that the reliability of the two-item measure of Social Networking Site Frequency was below the typically accepted threshold of Cronbach's a $=.70$ ( $a$ $=.64)$. These items were used together in the current study because of their conceptual importance as indicators of the frequency of social networking site use and were moderately correlated.

Consistent with the limited previous research, higher exposure to peers' alcohol-related posts on social media was associated with more frequent alcohol consumption by adolescents, even when controlling for offline peer alcohol norms. In controlling for perceptions of peer drinking in face-to-face settings, this study offers important new insight into how individual attributes interact with digital content. This study has thus highlighted the potential for vulnerability toward underage adolescent drinking that stems from exposure to peer alcohol use on social networking sites. By testing individual differences, we have demonstrated that particular adolescents are more sensitive than others to viewing alcohol-related content online. In an extension of previous research, the relationship between social networking site alcohol exposure and adolescent alcohol consumption was significantly moderated by the personality trait of excitement seeking. Adolescents higher in excitement seeking appeared to be more susceptible to online alcohol consumption norms. The findings of the present study have highlighted the value of investigating individual differences when considering online alcohol content and drinking behavior. Such potential vulnerabilities deserve consideration with respect to the role of media consumption in development, as adolescent drinking has well-documented detrimental consequences for immediate and longerterm health and well-being.

\section{Footnotes}

1. We additionally conducted a three-way interaction analysis following the same steps outlined above, including the interaction term, "Alcohol Exposure $\times$ Conscientiousness $\times$ Excitement Seeking" at Step 4. The interaction term was not significant, $F_{\text {chg }}(1,807)=0.03, p=.864$. We removed it to retain a more parsimonious model.

\section{Acknowledgements}

The Youth Activity Participation Study of Western Australia was funded by grants under Australian Research Council's Discovery Projects funding scheme: DP0774125 and DP1095791 to Bonnie Barber and Jacquelynne Eccles, and DP130104670 to Bonnie Barber, Kathryn Modecki, and Jacquelynne Eccles. The authors would like to thank Kathryn Modecki and the YAPS-WA research team, and extend additional thanks to all schools, staff, and participants involved in the YAPS-WA study. We also thank Alexander W. O'Donnell for contributions to the analysis.

\section{References}

Anderson, M., \& Jiang, J. (2018). Teens' social media habits and experiences. Pew Research Centre. https://www.pewinternet.org/2018/11/28/teens-social-media-habits-and-experiences/

Australian Communications and Media Authority. (2013). Like, post, share: Young Australians' experience of social media. https://apo.org.au/sites/default/files/resource-files/2013-08/apo-nid35223.pdf

Australian Institute of Health and Welfare. (2011) Young Australians: Their health and wellbeing 2011. https://www.aihw.gov.au/getmedia/14eed34e-2e0f-441d-88cb-ef376196f587/12750.pdf.aspx?inline=true

Bekman, N. M., Cummins, K., \& Brown, S. A. (2010). Affective and personality risk and cognitive mediators of initial adolescent alcohol use. Journal of Studies on Alcohol and Drugs, 71(4), 570-580.

https://doi.org/10.15288/jsad.2010.71.570 
Beullens, K., \& Schepers, A. (2013). Display of alcohol use on Facebook: A content analysis. Cyperpsychology, Behavior, and Social Networking, 16(7), 497-503. https://doi.org/10.1089/cyber.2013.0044

Beullens, K., \& Vandenbosch, L. (2016). A conditional process analysis on the relationship between the use of social networking sites, attitudes, peer norms, and adolescents' intentions to consume alcohol. Media Psychology, 19(2), 310-333. https://doi.org/10.1080/15213269.2015.1049275

Bogg, T., \& Roberts, B. W. (2004). Conscientiousness and health-related behaviors: A meta-analysis of the leading behavioral contributors to mortality. Psychological Bulletin, 130(6), 887-919. https://doi.org/10.1037/00332909.130.6.887

Borsari, B., \& Carey, K. B. (2001). Peer influences on college drinking: A review of the research. Journal of Substance Abuse, 13(4), 391-424. https://doi.org/10.1016/S0899-3289(01)00098-0

Boyle, G. J., Matthews, G., \& Saklofske, D. H. (2008). The SAGE handbook of personality theory and assessment: Personality measurement and testing (Vol. 2). SAGE Publications. https://doi.org/10.4135/9781849200479

Boyle, S. C., LaBrie, J. W., Froidevaux, N. M., \& Witkovic, Y. D. (2016). Different digital paths to the keg? How exposure to peers' alcohol-related social media content influences drinking among male and female first-year college students. Addictive Behaviors, 57, 21-29. https://doi.org/10.1016/j.addbeh.2016.01.011

Chou, H-T. G., \& Edge, N. (2012). “They are happier and having better lives than I am": The impact of using Facebook on perceptions of others' lives. Cyberpsychology, Behavior, and Social Networking, 15(2), 117-121. https://doi.org/10.1089/cyber.2011.0324

Clapp, J. D., \& McDonnell, A. L. (2000). The relationship of perceptions of alcohol promotion and peer drinking norms to alcohol problems reported by college students. Journal of College Student Development, 41(1), 19-26.

Costa, P. T., Jr., \& McCrae, R. R. (1992). Revised NEO Personality Inventory (NEO-PI-R) and NEO Five-Factor Inventory (NEO-FFI) professional manual. Psychological Assessment Resources.

De Fruyt, F., Mervielde, I., Hoekstra, H. A., \& Rolland, J.-P. (2000). Assessing adolescents' personality with the NEOPI-R. Assessment, 7(4), 329-345. https://doi.org/10.1177/107319110000700403

Drane, C. F., Modecki, K. L., \& Barber, B. L. (2017). Disentangling development of sensation seeking, risky peer affiliation, and binge drinking in adolescent sport. Addictive Behaviors, 66, 60-65.

https://doi.org/10.1016/j.addbeh.2016.11.001

Egan, K. G., \& Moreno, M. A. (2011). Alcohol references on undergraduate males' Facebook profiles. American Journal of Men's Health, 5(5), 413-420. https://doi.org/10.1177/1557988310394341

Ellison, N. B., Steinfield, C., \& Lampe, C. (2007). The benefits of Facebook "friends": Social capital and college students' use of online social network sites. Journal of Computer-Mediated Communication, 12(4), 1143-1168. https://doi.org/10.1111/j.1083-6101.2007.00367.x

Elmore, K. C., Scull, T. M., \& Kupersmidt, J. B. (2016). Media as a "super peer": How adolescents interpret media messages predicts their perception of alcohol and tobacco use norms. Journal of Youth and Adolescence, 46(2), 376-387. https://doi.org/10.1007/s10964-016-0609-9

Fournier, A. K., \& Clarke, S. W. (2011). Do college students use Facebook to communicate about alcohol? An analysis of student profile pages. Cyberpsychology: Journal of Psychosocial Research on Cyberspace, 5(2), Article 2. https://cyberpsychology.eu/article/view/4246/3292 
Fredricks, J. A., \& Eccles, J. S. (2005). Developmental benefits of extracurricular involvement: Do peer characteristics mediate the link between activities and youth outcomes? Journal of Youth and Adolescence, 34(6), 507-520. https://doi.org/10.1007/s10964-005-8933-5

Geusens, F., \& Beullens, K. (2017). The reciprocal associations between sharing alcohol references on social networking sites and binge drinking: A longitudinal study among late adolescents. Computers in Human Behavior, 73, 499-506. https://doi.org/10.1016/j.chb.2017.03.062

Geusens, F., Vangeel, J., Vervoort, L., Van Lippevelde, W., \& Beullens, K. (2019). Disposition-content congruency in adolescents' alcohol-related social media (self-) effects: The role of the five-factor model. Journal of Studies on Alcohol and Drugs, 80(6), 631-640. https://doi.org/10.15288/jsad.2019.80.631

Glazer, E., Smith, S. W., Atkin, C., \& Hamel, L. M. (2010). The effects of sensation seeking, misperceptions of peer consumption, and believability of social norms messages on alcohol consumption. Journal of Health Communication, 15(8), 825-839. https://doi.org/10.1080/10810730.2010.522222

Guerin, N. \& White, V. (2018). ASSAD 2017: Statistics \& trends: Australian secondary students' use of tobacco, alcohol, over-the-counter drugs, and illicit substances. Cancer Council Victoria.

https://www.health.gov.au/sites/default/files/secondary-school-students-use-of-tobacco-alcohol-and-otherdrugs-in-2017_1.pdf

Hagger-Johnson, G., Bewick, B. M., Conner, M., O'Connor, D., \& Shickle, D. (2012). School-related conscientiousness, alcohol drinking, and cigarette smoking in a representative sample of English school pupils. British Journal of Health Psychology, 17(3), 644-665. https://doi.org/10.1111/j.2044-8287.2011.02061.x

Haines, M., \& Spear, S. F. (1996). Changing the perception of the norm: A strategy to decrease binge drinking among college students. Journal of American College Health, 45(3), 134-140.

https://doi.org/10.1080/07448481.1996.9936873

Hayes, A. F. (2013). Introduction to mediation, moderation, and conditional process analysis: A regression-based approach. The Guilford Press.

Hong, R. Y., \& Paunonen, S. V. (2009). Personality traits and health risk behaviors in university students. European Journal of Personality, 23(8), 675-696. https://doi.org/10.1002/per.736

Huang, G. C., Unger, J. B., Soto, D., Fujimoto, K., Pentz, M. A., Jordan-Marsh, M., \& Valente, T. W. (2014). Peer influences: The impact of online and offline friendship networks on adolescent smoking and alcohol use. Journal of Adolescent Health, 54(5), 508-514. https://doi.org/10.1016/j.jadohealth.2013.07.001

Hustad, J. T. P., Pearson, M. R., Neighbors, C., \& Borsari, B. (2014). The role of alcohol perceptions as mediators between personality and alcohol-related outcomes among incoming college-student drinkers. Psychology of Addictive Behaviors, 28(2), 336-347. https://doi.org/10.1037/a0033785

Kandel, D. B., \& Andrews, K. (1987). Processes of adolescent socialization by parents and peers. International Journal of the Addictions, 22(4), 319-342. https://doi.org/10.3109/10826088709027433

Kann, L., McManus, T., Harris, W. A., Shanklin, S. L., Flint, K. H., Queen, B., Lowry, R., Chyen, D., Whittle, L., Thornton, J., Lim, C., Bradford, D., Yamakawa, Y., Leon, M., Brener, N., \& Ethier, K. A. (2018). Youth risk behavior surveillance - United States, 2017 (MMWR Surveillance Summaries, Vol. 67, No. 8). Centers for Disease Control and Prevention. https://www.cdc.gov/healthyyouth/data/yrbs/pdf/2017/ss6708.pdf

Kim, J.-H., \& Kim, K. S. (2012). The role of sensation seeking, perceived peer pressure, and harmful alcohol use in riding with an alcohol-impaired driver. Accident Analysis \& Prevention, 48, 326-334.

https://doi.org/10.1016/j.aap.2012.01.033 
Klimstra, T. (2013). Adolescent personality development and identity formation. Child Development Perspectives, 7(2), 80-84. https://doi.org/10.1111/cdep.12017

Lenhart, A. (2015). Teens, social media and technology overview 2015. Pew Research Center. https://www.pewresearch.org/internet/2015/04/09/teens-social-media-technology-2015/

Lenhart, A., \& Madden, M. (2007). Teens, privacy and online social networks. Pew Research Center. http://www.pewinternet.org/2007/04/18/teens-privacy-and-online-social-networks/

Litt, D. M., \& Stock, M. L. (2011). Adolescent alcohol-related risk cognitions: The roles of social norms and social networking sites. Psychology of Addictive Behaviors, 25(4), 708-713. https://doi.org/10.1037/a0024226

Marino, C., Vieno, A., Pastore, M., Albery, I. P., Frings, D., \& Spada, M. M. (2016). Modelling the contribution of personality, social identity and social norms to problematic Facebook use in adolescents. Addictive Behaviors, 63, 51-56. https://doi.org/10.1016/j.addbeh.2016.07.001

McCabe, K. O., Modecki, K. L., \& Barber, B. L. (2016). Participation in organized activities protects against adolescents' risky substance use, even beyond development in conscientiousness. Journal of Youth and Adolescence, 45(11), 2292-2306. https://doi.org/10.1007/s10964-016-0454-x

McCrae, R. R., \& John, O. P. (1992). An introduction to the five-factor model and its applications. Journal of Personality, 60(2), 175-215. https://doi.org/10.1111/j.1467-6494.1992.tb00970.x

Modecki, K. L., Barber, B. L., \& Eccles, J. S. (2014). Binge drinking trajectories across adolescence: For early maturing youth, extra-curricular activities are protective. Journal of Adolescent Health, 54(1), 61-66.

https://doi.org/10.1016/j.jadohealth.2013.07.032

Neal, D. J., \& Simons, J. S. (2007). Inference in regression models of heavily skewed alcohol use data: A comparison of ordinary least squares, generalized linear models, and bootstrap resampling. Psychology of Addictive Behaviors, 21(4), 441-452. https://doi.org/10.1037/0893-164X.21.4.441

Pegg, K. J., O'Donnell, A. W., Lala, G., \& Barber, B. L. (2018). The role of online social identity in the relationship between alcohol-related content on social networking sites and adolescent alcohol use. Cyberpsychology, Behavior, and Social Networking, 21(1), 50-55. https://doi.org/10.1089/cyber.2016.0665

Raynor, D. A., \& Levine, H. (2009). Associations between the five-factor model of personality and health behaviors among college students. Journal of American College Health, 58(1), 73-82.

https://doi.org/10.3200/JACH.58.1.73-82

Rimal, R. N., \& Real, K. (2005). How behaviors are influenced by perceived norms: A test of the theory of normative social behavior. Communication Research, 32(3), 389-414. https://doi.org/10.1177/0093650205275385

Schroeder, C. M., \& Prentice, D. A. (1998). Exposing pluralistic ignorance to reduce alcohol use among college students. Journal of Applied Social Psychology, 28(23), 2150-2180. https://doi.org/10.1111/j.1559-

1816.1998.tb01365.x

Stattin, H., Gustafson, S. B., \& Magnusson, D. (1989). Peer influences on adolescent drinking: A social transition perspective. Journal of Early Adolescence, 9(3), 227-246. https://doi.org/10.1177/0272431689093004

Stoddard, S. A., Bauermeister, J. A., Gordon-Messer, D., Johns, M., \& Zimmerman, M. A. (2012). Permissive norms and young adults' alcohol and marijuana use: The role of online communities. Journal of Studies on Alcohol and Drugs, 73(6), 968-975. https://doi.org/10.15288/jsad.2012.73.968

Valkenburg, P. M., \& Peter, J. (2013). The differential susceptibility to media effects model. Journal of Communication, 63(2), 221-243. https://doi.org/10.1111/jcom.12024 
Vernon, L., Modecki, K. L., \& Barber, B. L. (2017). Tracking effects of problematic social networking on adolescent psychopathology: The mediating role of sleep disruption. Journal of Clinical Child and Adolescent Psychology, 46(2), 269-283. https://doi.org/10.1080/15374416.2016.1188702

Wilson, K., Fornasier, S., \& White, K. M. (2010). Psychological predictors of young adults' use of social networking sites. Cyberpsychology, Behavior, and Social Networking, 13(2), 173-177. https://doi.org/10.1089/cyber.2009.0094

\section{Correspondence to:}

Riley Anne Scott

Gold Coast Campus, School of Applied Psychology

Level 7 Health Building (G40), Parklands Drive

Southport QLD 4215

Australia

Email: riley.scott(at)griffithuni.edu.au

Editorial record: First submission received on October 16, 2019. Revisions received on July 17, 2020 and October 9, 2020. Accepted for publication on October 20, 2020.

Editor in charge: Kristian Daneback

\section{About Authors}

Riley A. Scott is a PhD Candidate in the Griffith University School of Applied Psychology. Her research interests focus on adolescent and young adults' intrapersonal characteristics and use of the Internet and social media, with a specific focus on the development and maintenance of close friendships, and how experiences unique to the digital or online environment affect how people think, feel and act.

Bonnie L. Barber (Ph.D., University of Michigan) is the Dean (Research) for the Health Group at Griffith University. Bonnie is a developmental psychologist specializing in youth engagement and well-being. She is especially interested in the impact of young people's leisure time activity pursuits and their use of technology. 